\title{
Circadian factors during sustained performance: Background and methodology
}

\author{
TIMOTHY H. MONK, JEFFREY E. FOOKSON, JACOB KREAM, MARGARET L. MOLINE, \\ CHARLES P. POLLAK, and MURIEL B. WEITZMAN \\ Cornell University Medical College, Westchester Division, The New York Hospital, White Plains, New York
}

\begin{abstract}
It is well established that there is a complex timekeeping mechanism in the human brain. This mechanism is associated with a variety of physiological and psychological rhythms having a period of about a day, and thus referred to as circadian rhythms. The circadian system has recently been modeled in terms of two underlying oscillators, one much more resistant to changes in routine than the other. These oscillators are considered to be endogenous, that is, internal to the organism, and not reliant for their existence upon changes in the person's environment or general behavior. They thus continue to run even when the sleep/wake cycle is suspended, as in sustained operations. Thus, by their very nature, sustained operations require the individual to override the inputs that are coming from his or her circadian system (especially the indication that sleep is required). The aim of this paper is to provide a background to the area of circadian rhythms research, including a section on the methodology, so that the impact of the circadian system on sustained operations can be better understood.
\end{abstract}

This paper has two purposes. The first is to discuss recent findings in the area of human circadian (approximately 24-h) rhythms, and their implication for sustained operations. The second is to describe the methodology that is used to study these rhythms in the laboratory, and uses as an example the particular techniques employed at the Institute of Chronobiology, Cornell University Medical College.

\section{Research Procedures in Chronobiology}

The study of circadian rhythms is a major part of the discipline of chronobiology, the study of temporal structure in all living organisms (Halberg, 1969). Since the discipline is young, much chronobiological research is still concerned with simply demonstrating that particular rhythms exist and describing the form that they take (Monk, 1982b). However, recent research into human circadian rhythms has been concerned with the more complex issues regarding mechanisms and implications of the circadian system (Wever, 1979). Physiological variables are often based upon an analysis of the blood (Weitzman, Czeisler, \& Moore-Ede, 1979) or urine (Lewis \& Lobban, 1956) of the subject, enabling the concentration of various hormones (e.g., cortisol, melatonin) or electrolytes (e.g., sodium, potassium) to be obtained. Body temperature is often taken, as are measures of heart rate and blood pressure (Wever, 1979). Sleep measures are determined polygraphically (Webb, 1982) and are categorized into sleep "stages" (Rechtschaffen \& Kales, 1968)

Supported by National Institutes of Health Grants MH 37814-02 and AG-04135-01 and NASA Co-operative Agreement No. NCC 2-253. The authors' mailing address is: Institute of Chronobiology, Department of Psychiatry, Comell University Medical College, Westchester Division, The New York Hospital, 21 Bloomingdale Road, White Plains, NY 10605. for analysis. Performance measures are taken by administering "performance tests" at particular times during the experiment. These tests are usually of comparatively short duration and reflect the speed or accuracy with which a task can be performed (Folkard \& Monk, 1983). Additionally, tests of mood and subjective activation can be associated with the performance variables (Froberg, 1977). Measures taken from all these variables are then plotted as functions of time, and the various patterns and interactions are studied, often with fairly complex statistical techniques (Monk, 1982b).

Human circadian rhythm investigation is thus interdisciplinary, involving psychologists, psychiatrists, physiologists, neuroendocrinologists, neurologists, and polysomnographers. Since this review is concerned primarily with the implications for sustained operations, there is a bias toward the psychological aspects. Other areas of investigation are of equal importance and are also discussed.

\section{Circadian Rhythms}

There are dramatic differences in human physiology and psychology from one time of day to another. The most obvious difference is that the individual is usually asleep and relatively immobile at night, and awake and active during the day. However, these differences represent a cyclic pattern that is considerably more complex than a simple alternation (Aschoff, 1981). Not only are there time-of-day differences within the waking interval, but there are regular changes in the patterning of sleep "type" within the period of sleep (Webb, 1982). Thus, circadian rhythms are not simply a step function between sleep and wakefulness, and are not simply a transition process between the two. Rather, they appear to result from the workings of some cyclic "biological clock" or internal timekeeping system. 
The most obvious proof for the existence of such a cyclic system is the fact that circadian rhythms are selfsustaining, even when the pattern of sleep and wakefulness is entirely absent. Thus, as part of a series of sustained operation studies, Froberg (1977) was able to demonstrate the maintenance of circadian rhythms even during $72 \mathrm{~h}$ of sleep deprivation, with activity, knowledge of clock time, and diet all rigidly controlled. Figures 1 and 2 illustrate the average body temperature and subjective alertness rhythms collected from 15 young females. Although there is an overall decreasing trend, it is quite clear that circadian rhythmicity persists in both physiological and psychological measures.

There is a limit to the length of time that an individual

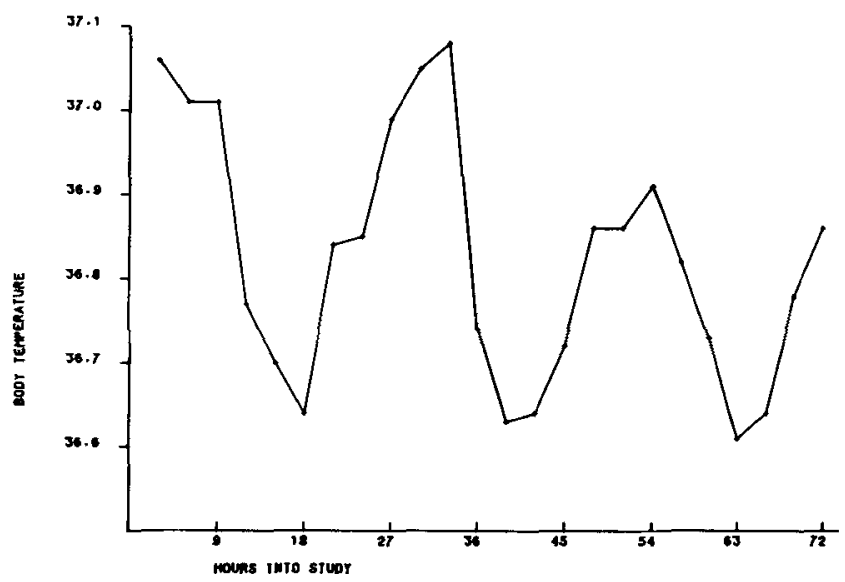

Figure 1. The average body temperature of 15 subjects experiencing $72 \mathrm{~h}$ of sleep deprivation under constant conditions (after Froberg, 1977).

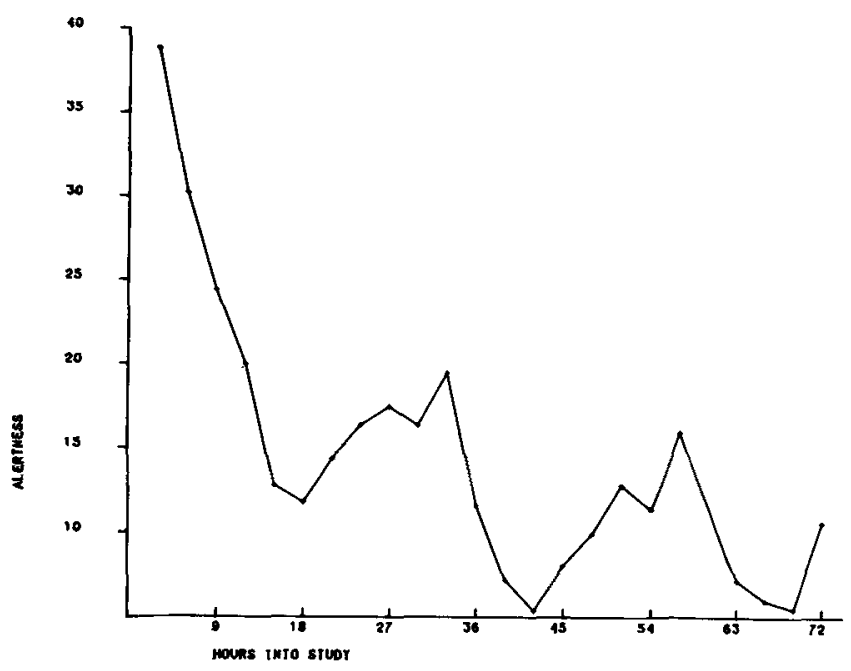

Figure 2. The average subjective alertness of 15 subjects experiencing $72 \mathrm{~h}$ of sleep deprivation under constant conditions (after Froberg, 1977).

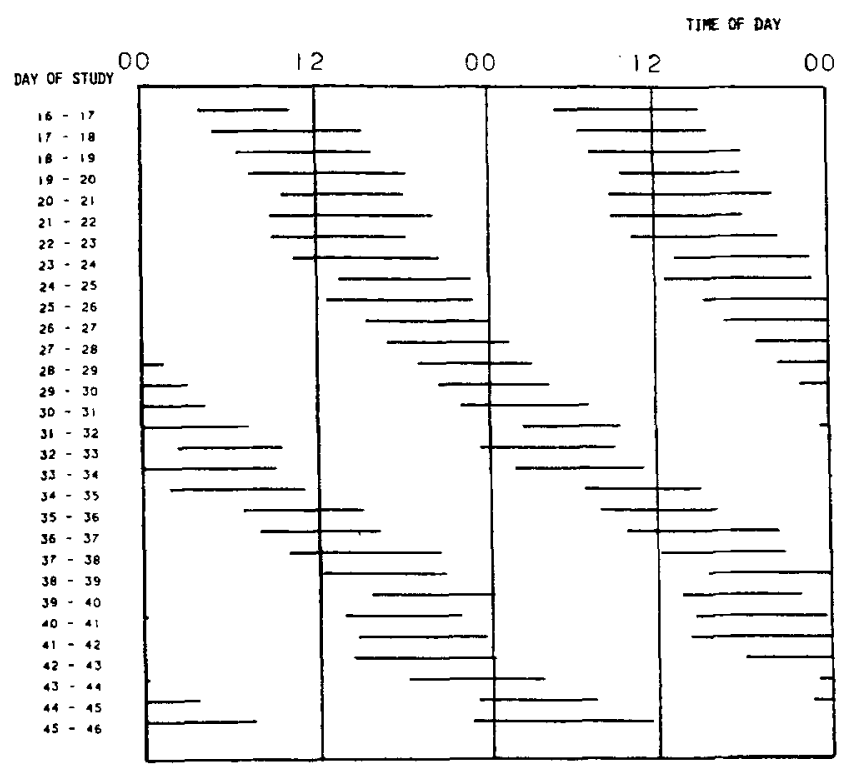

Figure 3. The sleep/wake cycle of a 23-year-old male subject experiencing 4 weeks of free-running under temporal isolation in the present laboratory. The solid line represents the time spent in bed (in darkness).

can be deprived of sleep, and thus a limit to the length of time for which his/her general behavior is held constant. Longer term time-isolation experiments must allow the subject to sleep. In many time-isolation studies, the experimenter dictates the sleep/wake schedule. However, when the subject him- or herself selects the timing of rest, activity, and meals, the study is referred to as a "freerunning" experiment. Such experiments were pioneered by Aschoff and Wever in Erling-Andechs, West Germany, in the 1960s (Wever, 1979). The major American freerunning studies have been carried out by Weitzman's group in New York (Weitzman et al., 1979). The most important finding from both centers is that for many individuals the circadian system is able to maintain its cyclic integrity, even during experiments lasting several months. Thus, the self-sustaining aspect of the circadian system remarked upon in reference to Froberg's (1977) 72-h study is present in much longer term experiments in which sleep is permitted.

A typical free-running result from our laboratory is illustrated in Figure 3, which shows the sleep/wake cycle of a 23-year-old man who spent 2 weeks free-running in temporal isolation. Time spent in bed (in darkness) is represented by the solid lines. Each day is plotted twice; a total of $48 \mathrm{~h}$ is represented on the " $\mathrm{x}$-axis." This allows the "drift" in period away from $24 \mathrm{~h}$ to become more apparent. "Day of study" runs down from the top along the "y-axis."

The fact that the free-running bars do not align immediately below each other indicates that the subject's freerunning "days" were not exactly $24 \mathrm{~h}$ in length. By fitting a straight line to the beginning of each sleep period, we can obtain an estimate of the period length of the sub- 
TIME OF DAY

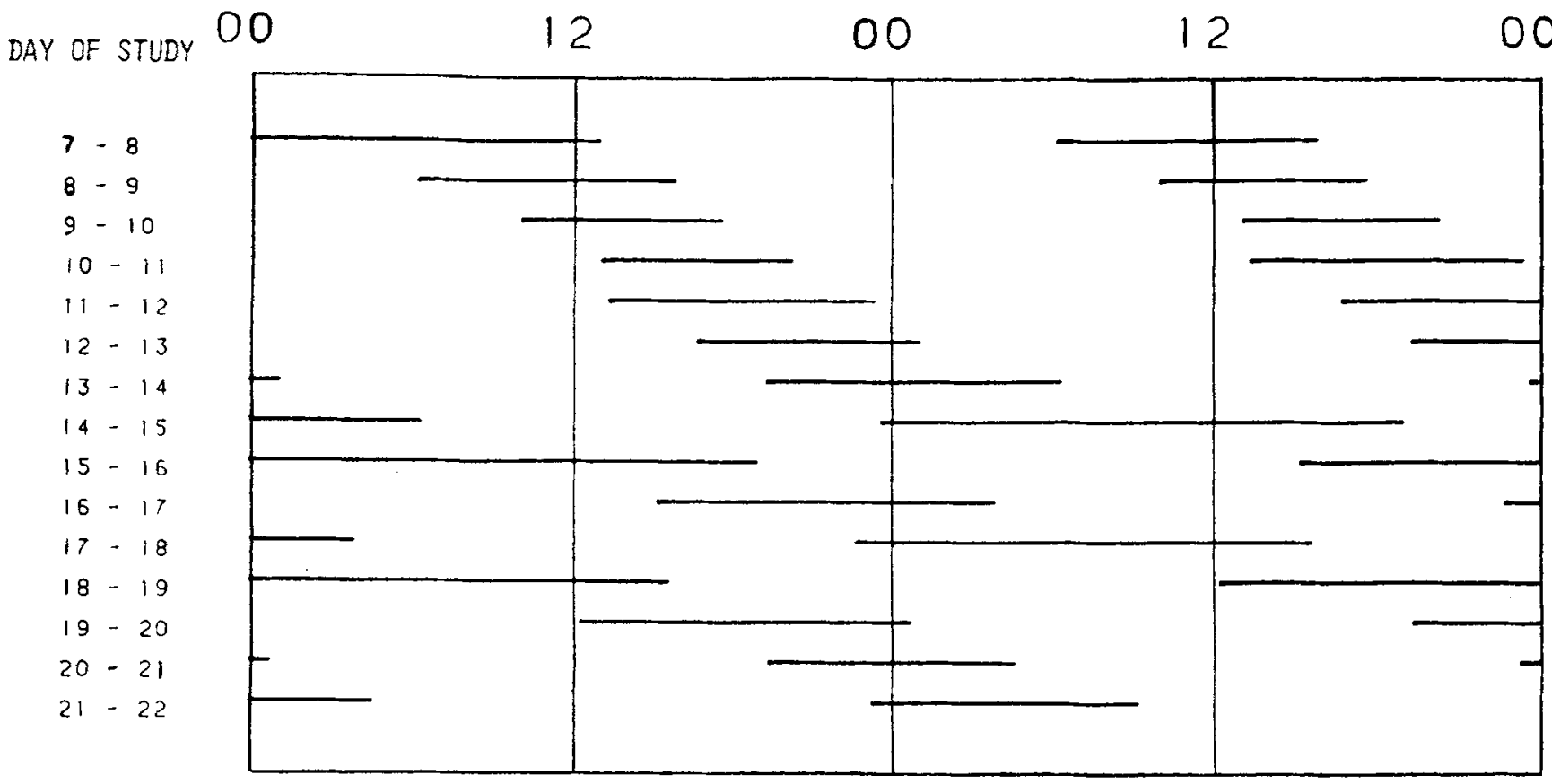

Figure 4: The sleep/wake cycle of a 56-year-old female subject experiencing 2 weeks of free-running under temporal isolation. The solid line represents the time spent in bed (in darkness).

ject's "day." In this example, the period length is $25 \mathrm{~h}$, a typical result for a young man (see Wever, 1979). The circadian system thus appears to "run slow" in general, and it is extremely rare to find a human free-running sleep/wake cycle that is shorter than $24 \mathrm{~h}$. Rectal temperatures were measured every minute. Periodogram analysis of this data indicated that the temperature rhythm also had a period of about $25 \mathrm{~h}$, in synchrony with that of the sleep/wake cycle.

Although the preceding discussion emphasizes the regularity and integrity of the circadian system, there are individuals and situations in which the sleep/wake cycle can appear to be very irregular, for example, with extremely long $(12-15-h)$ sleep periods mixed with sleep periods of more normal length. This is referred to as "spontaneous desynchronization" (Wever, 1975), and it often occurs toward the end of an otherwise regular freerunning episode. An illustration of this, in a 56-year-old woman, is given in Figure 4. During the latter part of the study, it is apparent that extremely long sleep periods were occurring, with a breakdown in the more typical free-running pattern that had occurred earlier.

Contrary to initial impressions, desynchronization does not represent a chaotic breakdown of the circadian system. Rather, it represents a situation in which the cycles of sleep/wake and temperature simply break apart, running at different periods, with the period of the sleep/wake cycle being considerably longer than that of the body tem- perature rhythm. During desynchronization, the two cycles no longer exhibit stable relationships to each other. The two still influence each other, however, with the duration of the sleep episode being governed by the temperature phase at sleep onset (Czeisler, Weitzman, MooreEde, \& Zimmerman, 1980). When the temperature at sleep onset is high and falling, long sleeps are experienced; when the temperature is low and rising, comparatively short sleeps occur. This influence is particularly important in sustained operations studies in which naps are permitted. As Moses, Lubin, Naitoh, and Johnson (1978) confirmed, there are some phases of the circadian system at which naps are considerably more restorative to the individual than at others. This is in accord with a relationship between temperature and sleep duration.

Desynchronization is particularly important in demonstrating that body temperature and sleep/wake cycles are under the control of two separate, but interactive, oscillators (or groups of oscillators). The oscillator governing the temperature rhythm is referred to as "Group I" (Wever, 1975) or " $X$ " (Kronauer, Czeisler, Pilato, Moore-Ede, \& Weitzman, 1982), and that responsible more for the sleep/wake cycle, as "Group II" or "Y." In contrast to the sleep/wake cycle, the temperature rhythm takes many days to adjust fully to a change in routine, and the Group I or " $X$ " oscillator is thus held to be the less labile of the two. In this article, we refer to 
the temperature oscillator as the "deep" oscillator, and to the one responsible for the sleep/wake cycle as the "labile" oscillator.

\section{Performance}

A major consideration in sustained operations studies is the performance efficiency of the individual. In circadian terms, it is thus important to know whether the deep or the labile oscillator is controlling circadian performance rhythms, or whether time-of-day differences simply reflect a "time since waking" effect. Interestingly, studies of diurnal variation in human performance predate the use of the terms "free-running" and "circadian" by about half a century. Early studies were concerned primarily with the scheduling of school timetables and used cognitive tasks such as memory for prose and mental arithmetic. Although their findings were not uniformly consistent (see Lavie, 1980, for an excellent review), Gates (1916) concluded that "in general, the forenoon is the best time for strictly mental work ... while the afternoon might best be taken up with school subjects in which motor factors are predominant"' (p. 149). Gate's conclusion is important, both in postulating a morning superiority in cognitive (mental) performance and in differentiating between cognitive and motor tasks. The afternoon inferiority in cognitive performance was held to result from a buildup in mental fatigue, and these early studies thus considered diurnal variation in performance to result from a "time since waking" effect. The differentiation between cognitive and simple repetitive tasks is particularly important when contemporary studies are considered.

A rather different approach to the study of circadian performance rhythms was pioneered by Kleitman (1963), who was particularly impressed by the parallelism that appeared to exist between the circadian variation in performance and that in body temperature. In contrast to the early school-timetabling studies, Kleitman favored tasks with a low cognitive load, such as card dealing and choice reaction. In a series of studies, Kleitman found that performance tended to follow the temperature rhythm, even when the two were in the process of phase adjustment to an acute change in routine. He concluded that there was a causal relationship between circadian variation in body temperature and performance.

In a later, much more extensive series of studies, Colquhoun and his associates (Colquhoun, Blake, \& Edwards, 1968a, 1968b, 1969) confirmed this finding, although they were careful to emphasize parallelism, rather than causality. Colquhoun's tasks were also mainly those requiring either the maintenance of vigilance or a simple throughput of information. Interestingly, when a more cognitive digit-span task was used, a very different time-of-day function emerged, with the performance peak occurring about $12 \mathrm{~h}$ earlier than the temperature peak (Blake, 1967). However, performance was still tied to the deep oscillator, using the mechanism of an "arousal rhythm." This rhythm was held to be parallel to the temperature rhythm and related to performance by an "inverted U". shaped function, which then explained the observed differences in diurnal performance rhythms (Hockey \& Colquhoun, 1972).

During the last 10 years, this notion of performance rhythm's being tied exclusively to the temperature rhythm (and thus the deep oscillator) has been cast in doubt. Folkard (1973) found a midday superiority in verbal reasoning performance, and later, Folkard, Knauth, Monk, and Rutenfranz (1976) radically affected the timeof-day function by systematically varying the memory load of a task. Neither of these results fits perfectly with the "'arousal rhythm' model (Monk, 1982a). More important in terms of oscillatory control was the finding that intertask differences in phase (time of peak) were also reflected in differences in the rate at which circadian performance rhythms phase-adjusted to a change in routine (Folkard \& Monk, 1982; Monk, Knauth, Folkard, \& Rutenfranz, 1978). Such rate differences are not explicable in terms of simple control by the deep oscillator.

Recently, groups both at Erling-Andechs (Folkard, Wever, \& Wildgruber, 1983) and in New York (Monk, Weitzman, Fookson, \& Moline, 1984; Monk, Weitzman, Fookson, Moline, Kronauer, \& Gander, 1983) have investigated the oscillator control aspect directly, by causing the two oscillators to run at different periods. Such experiments revealed that, although simple repetitive (e.g., motor) tasks are indeed under deep oscillator control, different processes are involved when more cognitive tasks are considered. Such processes can involve either shorter circadian periodicities (Folkard et al., 1983; Monk et al., 1984) or simply a strong measure of control from the more labile sleep/wake oscillator (Monk et al., 1983).

These contemporary findings regarding the mechanisms underlying circadian performance rhythmicity have important ramifications in the sustained performance situation. Although one can still use the temperature rhythm as a predictor of performance on the more simple repetitive and vigilance types of task, it is likely to be of limited use when the more cognitive tasks are considered. At present, we cannot predict with certainty how performance on such tasks will behave. It is quite possible, however, as Folkard and Monk (1982) found in a reanalysis of Froberg's (1977) data, that short period (21-23-h) circadian fluctuations might appear.

In conclusion, recent research on the circadian system has strongly influenced the way in which sustained performance is considered. Clearly, the notion of a continuous decline in abilities over the vigil must be abandoned, and circadian inputs to sleep, physiology, and performance must be recognized. The second part of this paper describes some of the techniques used to conduct such research.

\section{METHOD}

\section{Facilities}

The methods and techniques that are to be described are used by the present authors and are based on those developed by the late Elliot $\mathrm{D}$. Weitzman and his former 
colleagues at Montefiore Hospital (Weitzman et al., 1979; Weitzman et al., 1982). The Laboratory of Human Chronophysiology (LHC) comprises our time-isolation apartments and related support facilities. Each subject has his or her own separate apartment. The apartments are constructed specifically to eliminate all sources of temporal information, including outside light and sound, through the use of double-entry doors to the suites, blocked windows, and sound-attenuating walls. Clocks, radios, televisions, and telephones are not permitted. Subjects are continuously monitored by video and audio techniques.

Unlike other time-isolation facilities (e.g., Wever, 1979), the LHC does not require that subjects be kept in social isolation. However, only the technical staff are allowed to interact regularly with the subjects, bringing them food, supervising performance tests, and talking socially to them as required. In order to avoid giving the the subjects any time cues, the technicians are instructed to make no reference to time in the presence of the subjects and are given random work shifts varying from 6 to $11 \mathrm{~h}$ in length. Visits to the subject by professional staff are scheduled to occur, whenever possible, soon after the subject's breakfast, whatever actual time of day that may be.

All food is prepared by the technical saff in a kitchen adjacent to the control room. Before the start of the study (see below), the subject is interviewed to determine his or her food preferences, and the necessary supplies are then purchased. No food or drink containing caffeine or alcohol is permitted. Subjects are encouraged to structure their eating behavior in terms of breakfast, lunch, dinner, and evening snack, although this is not enforced in free-running studies. Decaffeinated coffee, soda, and herbal tea are available on request on an ad-lib basis, as are small snacks (e.g., cookies). All such events are logged for later analysis.

Subject selection and recruitment constitute a significant part of the research effort. LHC subjects can be men or women, and have ranged in age from 14 to 83 years. Many are normal, healthy, paid volunteers, but patients suffering from various psychiatric, neurological, and sleep-related disorders have also been studied.

When a prospective subject contacts the Institute, the Recruitment Coordinator conducts an extensive telephone interview. The candidate receives brochures, a description of the study, and various questionnaires. Subjects who are then deemed suitable are brought in for a full day of medical and psychological screening involving social workers, physicians, clinical psychologists, and neuropsychologists. They also have interviews with the experimental psychologist and the Laboratory supervisor, and are given a demonstration of the computer system. During the study, the Recruitment Coordinator visits the subjects, maintains contact with the families if necessary, and provides consultation to the technical staff on subject management.

\section{Data Collection}

LHC protocols require the collection of large amounts of time-series data. A major part of this data collection (about 3,000 values per subject per day) is managed by a Digital Equipment Corporation VT-103 microcomputer located in each time-isolation apartment. These computers are used to collect body temperatures every minute from a rectal probe, to allow events to be recorded (meals, showers, exercise, etc.), and to administer tests for assessing a subject's cognitive and affective state. Each VT103 has a dual TU-58 block-addressable tape drive for program and data storage and is connected to a printer (Epson MX-80) located in the control room, which allows the staff to monitor ongoing data collection (each minute, the minute and rectal temperature; each event, the event and the minute; details of each alertness test; and a summary of mood and performance scores). Every few days, the TU-58 tapes are removed, and the data are transferred to the VAX-750 mainframe computer for longterm storage and analysis.

\section{Computerized Data Collection}

The interactive part of the program consists of a set of "event menus," the software driver for managing them, and the performance test routines. Each menu is a numbered list of activities whose occurrence and timing we wish to record. To request an activity, the subject enters the appropriate number. A code representing the particular event and the current time is then recorded on the data tape. The same information, along with a narrative description of the event, is also recorded on the controlroom printer.

\section{Performance and Mood Assessment}

Subjective alertness. Psychological variables are measured at brief (and fairly frequent) "Alertness Tests," and at rather longer "Performance Sessions"' (described below), at which mood is also assessed. "Alertness tests" are given three times per hour, on average, and they evaluate subjective alertness, that is, subjective activation, or vigilance. This is measured with a standard Visual Analogue Scale (VAS) technique. The subject is presented with a line representing the extremes of his or her normal range of feelings, from "very sleepy" to "very alert." The subject is asked to place a marker somewhere along the line to indicate his or her feelings on the "sleepyalert" dimension at that particular time. The rationale for using the VAS techniques is discussed below in the "Performance Sessions" section. The VAS is presented on a computer terminal, with a flashing cursor being moved along a 10-cm line by means of two directional keys on the keyboard.

Performance sessions. Other aspects of mood and performance are measured much less frequently. In scheduling these performance sessions, it is important that the experimenters do not provide temporal information. The sessions are therefore tied to particular activities: waketime, bedtime, after urine collection, and every time solid food (meal or snack) is requested or scheduled. To avoid an excess of performance sessions, no session is given if one has occurred within the preceding $60 \mathrm{~min}$. Perfor- 
mance sessions are not given during a sleep period. Typically, about six or seven performance sessions occur per day. In addition to nine measures of mood (see below), three different aspects of performance efficiency are measured: manual dexterity, serial search, and complex verbal reasoning. These are discussed in detail below.

The three performance tests are given "on-line," using the VT-103 computer with specially connected "yes" and "no" buttons. The buttons are set into rubber cylinders that can be held comfortably in each hand. Responses are made by pressing the button with the thumb; releasing the button initiates the next trial. The three tasks are given in the order: search, verbal reasoning, manual dexterity. In each task, the subject is asked to respond as fast and as accurately as possible. A technician is always present in the room during performance sessions. Between test sessions, subjects are free to pursue hobbies, etc., and are actively encouraged to bring with them into the apartment plenty of material for this purpose.

Search task. The serial search task has a circadian performance rhythm in speed that is remarkably parallel to the circadian temperature rhythm (Monk, 1979). It is a modification of a task developed by Fort and Mills (1976) and later used by Monk and Leng (1982). Essentially, the task involves a simple "throughput" of information (Hockey \& Colquhoun, 1972), with a low "working memory" load, and an emphasis on simple perception and decision processes.

A line of 30 random uppercase letters of the alphabet is presented on the computer screen, and the subject is required to scan through the line from left to right and to search for an occurrence of the letter " $E$." The "yes" or the "no" button is then pressed to indicate whether or not an " $E$ " has been found. Thirty-two trials are given at each session; in half, no $E$ is present. On each trial, the latency from onset of the display to the initiation of a response is recorded. The accuracy of this response and the position of the " $E$ "' in the line are also recorded. This makes it possible for more sophisticated analyses (e.g., of scanning rate) to be made. After the 8th trial, the computer program automatically monitors the accuracy of the subject's responses and requires a restart if the accuracy rate falls below $75 \%$. This procedure ensures that subjects are genuinely able to do the task properly and that subjects who may have lost the motivation to perform the task do not simply press buttons at random. At the end of the 32 trials, the cumulative time taken and the percentage of correct responses are displayed on the screen. This feedback helps maintain the motivation of the subjects.

Verbal reasoning task. The complex verbal reasoning task is a modified form of the Baddeley Reasoning Test (Baddeley, 1968). This task has been shown to result in a reliable circadian performance rhythm peaking 4-6 h earlier than the rhythms of the more simple repetitive tasks (Folkard, 1975), and to show a faster phase adjustment to a change in routine (Hughes \& Folkard, 1976). To reduce visual confusion and the tendency of subjects to stereotype the ordering of the two letters, the letters " $M$ " and " $C$ " replace the " $A$ " and " $B$ " used by Baddeley and Folkard. The task requires subjects to respond to a randomly shuffled set of $\mathbf{3 2}$ sentences, each with a letter pair it is purporting to describe (e.g., "M IS NOT PRECEDED BY C-MC'; "C FOLLOWS M-CM"). The subject's task is to indicate whether or not the sentence is a true description of its letter pair. The sentences are in active and passive voice, are stated positively and negatively, and contain both possible orderings of the letters " $M$ " and " $C$." Half require a "yes" answer, and half a "no." The data-recording and error-checking procedures are the same as those for the serial search task.

Manual dexterity task. The manual dexterity task is a modified version of the Purdue Pegboard (Model 32020, Lafayette Instrument Co., Lafayette, IN) task. We determine the time taken to fill one row of 25 holes with the small metal pegs. Signals are sent to the computer when the first and last holes are filled, and the time taken is recorded. One run is performed with the dominant hand, and the second run with the nondominant hand. To provide feedback to the subject, the time taken is presented on the computer terminal.

The circadian performance rhythms of manual dexterity tasks seem to be controlled largely by the same underlying brain oscillator that controls the temperature rhythm (Monk, Weitzman, et al., 1983), although there is some evidence that performance on this task can peak earlier in the day (Monk \& Leng, 1982).

Mood. A VAS technique is used to take nine measures of mood at the start of each performance session. This technique is preferred because of the large number of test sessions given to subjects (usually several hundred per study), which makes the use of more time-consuming mood adjective check lists (e.g., Thayer, 1978) inadvisable because of subject resistance (leading to noncompliance and/or response stereotyping). The VAS technique has been used widely in circadian rhythms research (e.g., Folkard, Glynn, \& Lloyd, 1976; Froberg, 1977; Monk, Leng, et al., 1983; Weitzman et al., 1982).

Eight of the scales are unipolar VASs that measure the degree to which a particular mood is felt. The questions are: (1) How alert do you feel? (2) How sad do you feel? (3) How tense do you feel? (4) How much of an effort is it to do anything? (5) How happy do you feel? (6) How weary do you feel? (7) How calm do you feel? (8) How sleepy do you feel? A $10-\mathrm{cm}$ line appears below each question; the label "very little"' appears at the left end of the line and "very much" at the right end. The ninth scale is a bipolar one measuring subjective well-being versus malaise. The question is phrased as "Overall, how do you feel?, with "very bad" and "very good" at the ends of the line. The questions are presented one at a time on the computer, and a cursor is moved by the subject to indicate his or her response on that dimension. Pressing the left-hand ("no") button causes the cursor to move left; pressing the right-hand ("yes") button causes it to move 
right. When the cursor is stationary on the line for $3 \mathrm{sec}$, a value corresponding to the distance of the cursor from the left end of the line (0-36 arbitray units) is recorded by the computer, a tone sounds, and the next scale is presented.

\section{Practice Effects}

To reduce the impact of practice effects, strenuous efforts are made to familiarize the subject with the mood and performance measures. During screening, the subject is given training sessions on the performance tasks and a detailed explanation of the mood measures. He or she also completes a booklet of 20 practice sessions at home before the study starts.

\section{Polysomnography and Sleep Scoring}

Sleep recordings (polysomnographs) are obtained from each subject for all sleep periods. As soon as a sleep period is requested by the subject (or scheduled by the protocol), a technician enters the apartment to assist in preparing the subject for sleep recording (Weitzman, Czeisler, Zimmerman, Ronda \& Knauer, 1982). Wires leading from the electrodes are plugged into a connector board connected directly to a polygraph in the control room. Two polygraphs (Grass Instrument Company, Model 78D, 14channel) in the control room record up to four subjects at a time. Recordings are made at a paper speed of $10 \mathrm{~mm} / \mathrm{sec}$, providing 30-sec epochs for sleep scoring according to the standard criteria (Rechtschaffen \& Kales, 1968). Sleep-stage data are then entered into the computer for analysis.

\section{Blood Sampling}

When an experimental protocol requires plasma hormone measurement, blood samples are obtained at multiple intervals from an indwelling intravenous catheter. Using a computer-generated random schedule, technicians collect samples about every 20 min around the clock. During the subject's "day," the technicians enter the apartment to draw the samples. During a sleep episode, a side porthole connects the bedroom to a blood-sampling station in the main corridor between the time-isolation suites. This porthole is large enough that the entire infusion/sampling apparatus can pass through without disassembly. During the last 2 years, more than 10 subjects have been studied in this way, each for a period of about 3 weeks. The apparatus and exact procedures were described in detail in a separate publication (Weitzman et al., 1982) and are not repeated here.

\section{Hormone Assays}

The LHC has its own biochemistry laboratory for the analysis of blood and urine samples. Radioimmunoassays are currently available for measurement of the following human peptide hormones: growth hormone, insulin, prolactin, follicle stimulating hormone, and luteinizing hormone. Steroid hormone assays are also available: cortisol, urinary free cortisol, 11-deoxycortisol, dexamethasone, progesterone, estrone, estradiol, estriol, dehydroi- soandrosterone and its sulfate, androsterone and its sulfate, testosterone, dihydrotestosterone, and androstendione.

\section{Current Protocols}

There are three major categories of protocol. All involve the complete isolation of the subject from all time cues. The first is the standard free-running protocol. For 5 days, the subject is entrained to a routine corresponding to his or her habitual sleep/wake cycle, as determined by a sleep diary kept for 2 weeks before the start of the study. This provides a baseline and ensures that the subject has a stable circadian system prior to the free-running section. At bedtime on the 5th night, the subject is told that, henceforth, all decisions regarding bedtime, waketime, and meals will be his/her own. This free-running portion is then continued for the remainder of the experiment (typically about 3 weeks). Unless called for in the protocol, napping is forbidden. Thus, subjects are prevented from lying on the bed and from sleeping in chairs during their (self-selected) period of wakefulness. If sleep is desired, the subject is required to change into night clothes, prepare for the polysomnograph electrodes, and get into bed. When naps are called for in the protocol, no distinction is made between naps and major sleeps; the only difference is that subjects are told that they can take a "daytime" nap.

The second category of protocol is referred to as the " "phase boundary," or "induction of insomnia," protocol An experiment for this protocol typically requires the subject to live in the time-isolation apartment for several months. Throughout the experiment, a strict schedule is enforced, with the timing of meals, snacks, bedtime, and waketime specified throughout by the experimenters. After a period of entrainment to the subject's standard 24-h routine, the day length is changed to be shorter or longer than $24 \mathrm{~h}$. Using this protocol, one can separate the underlying circadian oscillators and, as a consequence, disrupt the subject's sleep.

The third category of protocol involves the study of the process of phase adjustment of the circadian system to an acute shift in routine. The protocol typically lasts 15 days, with the subject being entrained to a 24-h routine throughout. After 5 days on his or her habitual routine, there is an acute shift in phase (typically of $6 \mathrm{~h}$ ), and the new schedule (of sleep and meal timings) is imposed for the remaining 10 days of the study.

Other protocols are usually of shorter duration and are specifically tailored to the subjects or patients being studied (e.g., a special 5-day protocol for Alzheimer's disease patients).

\section{CONCLUSIONS}

By their very definition, sustained operations require the individual to override his/her circadian system and to overcome the impulse to sleep. That impulse is the product of an extremely complex endogenous cyclic timekeeping system that also plays a large role in shap- 
ing mood and performance efficiency. Only by understanding the mechanisms of the circadian system can we get a true understanding of what the adverse effects of sustained operations are likely to be and how these effects can be ameliorated.

It is not easy to study the human circadian system. Studies are lengthy and require a large investment in laboratory and human resources. Furthermore, interdisciplinary teams of investigators are needed, in order to properly study all the various facets of the circadian system. Such efforts are, however, worthwhile, since an understanding of the mechanisms underlying a process as important and dramatic as the sleep/wake cycle has the potential for enormous theoretical and practical benefits.

\section{REFERENCES}

AsCHOFF, J. (Ed.). (1981). Handbook of behavioral neurobiology (Vol. 4). New York: Plenum Press.

BADDELEY, A. D. (1968). A 3-min reasoning test based on grammatical transformation. Psychonomic Science, 10, 341-342.

BLAKE, M. J. F. (1967). Time of day effects on performance in a range of tasks. Psychonomic Science, 9, 349-350.

Colquhoun, W. P., Blake, M. J. F., \& Edwards, R. S. (1968a). Experimental studies of shift word I: A comparison of 'rotating' and 'stabilised' 4-hour shift systems. Ergonomics, 11, 437-453.

Colquhoun, W. P., Blake, M. J. F., \& Edwards, R. S. (1968b). Experimental studies of shift work II: Stabilised 8-hour shift systems. Ergonomics, 11, 527-546.

Colquhoun, W. P., Blake, M. J. F., \& Edwards, R. S. (1969). Experimental studies of shift work III: Stabilised 12-hour shift systems. Ergonomics, 12, 865-882.

Czeisler, C. A., Weitzman, E. D., Moore-Ede, M. C., ZimmerMAN, J. C. (1980). Human sleep: Its duration and organisation depend on its circadian phase. Science, 210, 1264-1267.

FolkARD, S. (1975). Diurnal variation in logical reasoning. British Journal of Psychology, 66, 1-8.

FolkaRD, S., GlynN, C. J., \& Lloyd, J. W. (1976). Diurnal variation and individual differences in the perception of intractable pain. Journal of Psychosomatic Research, 20, 289-301.

Folkard, S., Knauth, P., Monk, T. H., \& Rutenfranz, J. (1976). The effect of memory load on the circadian variation in performance efficiency under a rapidly rotating shift system. Ergonomics, 19, 479-488.

FolkarD, S., \& Monk, T. H. (1982). Circadian rhythms in performance-one or more oscillators? In R. Sinz \& M. R. Rosenzwig (Eds.), Psychophysiology 1980 (pp. 541-548). New York: Elsevier/North Holland Press.

FolkaRD, S., \& MoNK, T. H. (1983). Chronopsychology: Circadian rhythms and human performance. In A. Gale \& J. Edwards (Eds.), Physiological correlates of human behaviour (Vol. 2, pp. 57-78). London: Academic Press.

Folkard, S., WeVer, R. A., \& Wildgruber, C. M. (1983). Multioscillatory control of circadian rhythms in human performance. Nature, 305, 223-226.

FORT, A., \& Mills, J. N. (1976). Der einfluss der tageszeit und des vorhergehenden schlaf-wach-musters auf die leistungsfahigkeit unmittelbar nach dem aufstehen. In G. Hildebrandt (Ed.), Biologische rhythmen und arbeit. Berlin: Springer-Verlag.

FroberG, J. E. (1977). Twenty-four-hour patterns in human performance, subjective and physiological variables and differences between morning and evening active subjects. Biological Psychology, 5, 119-134.

GATES, A. I. (1916). Variations in efficiency during the day, together with practice effects, sex differences, and correlations. University of California Publications in Psychology, 2, 1-156.
Halberg, F. (1969). Chronobiology. Annual Review of Physiology, 31, 675-725.

Hockey, G. R. J., \& Colquhoun, W. P. (1972). Diurnal variation in human performance: A review. In W. P. Colquhoun (Ed.), Aspects of human efficiency-diurnal rhythm and loss of sleep (pp. 39-107). London: English Universities Press.

Hughes, D. G., \& FolKARD, S. (1976). Adaptation to an 8-hour shift in living routine by members of a socially isolated community. $\mathrm{Na}$ ture, 264, 432-434.

Kleitman, N. (1963). Sleep and wakefulness (rev. ed.) Chicago: University of Chicago Press.

Kronauer, R. E., Czeisler, C. A., Pilato, S. F., Moore-Ede, M. C., \& Weitzman, E. D. (1982). Mathematical model of the human circadian system with two interacting oscillators. American Journal of Physiology, 242, R3-R17.

LAVIE, P. (1980). The search for cycles in mental performance from Lombard to Kleitman. Chronobiologia, 7, 247-256.

LEWIS, P. R., \& LoBBAN, M. C. (1956). Patterns of electrolyte excretion in human subjects during a prolonged period of life on a 22-hour day. Journal of Physiology, 133, 670-680.

Monk, T. H. (1979). Temporal effects in visual search. In J. N. Clare \& M. A. Sinclair (Eds.), Search and the human observer (pp. 3039). London: Taylor and Francis.

MonK, T. H. (1982a). The arousal model of time of day effects in human performance efficiency. Chronobiologia, 9, 49-54.

Monk, T. H. (1982b). Research methods of chronobiology. In W. B. Webb (Ed.), Biological rhythms, sleep and performance (pp. 27-57). New York: Wiley.

Monk, T. H., Knauth, P., Folkard, S., \& Rutenfranz, J. (1978). Memory-based performance measure in studies of shiftwork. Ergonomics, 21, 819-826.

Monk, T. H., \& LENG, V. C. (1982). Time of day effects in simple repetitive tasks: Some possible mechanisms. Acta Psychologica, 51, 207-221.

Monk, T. H., Leng, V. C., Folkard, S., \& Weitzman, E. D. (1983). Circadian rhythms in subjective alertness and core body temperature. Chronobiologia, 10, 49-55.

Monk, T. H., Weitzman, E. D., Fookson, J. E. Moline, M. L., KronaueR, R. E., \& GANDER, P. H. (1983). Task variables determine which biological clock controls circadian rhythms in human performance. Nature, 304, 543-545.

Monk, T. H., Weitzman, E. D., Fookson, J. E., \& Moline, M. L. (1984). Circadian rhythms in human performance efficiency under free-running conditions. Chronobiologia, 11, 343-354.

Moses, J., Lubin, A., Naitoh, P., \& Johnson, L. C. (1978). Circadian variation in performance, subjective sleepiness, sleep and oral temperature during an altered sleep-wake schedule. Biological Psychology, 6, 301-308.

RechtSChAFFEN, A., \& Kales, A. (Eds.). (1968). A manual of standardized terminology, techniques and scoring system for sleep stages of human subjects. Los Angeles: UCLA Brain Information Service.

THAYER, R. E. (1978). Towards a psychological theory of multidimensional activation (arousal). Motivation and Emotion, 2, 1-35.

WEBB, W. B. (Ed.). (1982). Biological rhythms, sleep and performance. New York: Wiley.

Weitzman, E. D., Czeisler, C. A., \& Moore-Ede, M. C. (1979). Sleep-wake, neuroendocrine and body temperature circadian rhythms under entrained and non-entrained (free-running) conditions in man. In M. Suda, O. Hayaishi, \& H. Nakagawa (Eds.), Biological rhythms and their central mechanisms (pp. 199-227). Amsterdam: Elsevier/North Holland Press.

Weitzman, E. D., Czeisler, C. A., Zimmerman, J. C., Ronda, J., \& KNAUER, R. S. (1982). Chronobiological disorders: Analytic and therapeutic techniques. In C. Guilleminault (Ed.), Disorders of sleeping and waking: Indications and techniques (pp. 297-329). Reading, MA: Addison-Wesley.

WeVER, R. (1975). The circadian multi-oscillator system of man. International Journal of Chronobiology, 3, 19-55.

WEVER, R. (1979). The circadian system of man: Results of experiments under temporal isolation. New York: Springer-Verlag. 